\title{
Mindful Self-Care for Caregivers: A Proof of Concept Study Investigating a Model for Embedded Caregiver Support in a Pediatric Setting
}

\author{
Katherine M. Bellone ${ }^{1,3} \cdot$ Stephen C. Elliott ${ }^{1,3} \cdot$ Linda S. Hynan $^{1,2} \cdot$ Beth Warren $^{3} \cdot$ Robin B. Jarrett $^{1}$
}

Accepted: 26 May 2021 / Published online: 9 June 2021

○) Springer Science+Business Media, LLC, part of Springer Nature 2021

\begin{abstract}
We conducted an open-trial proof of concept study to determine the safety, acceptability, and feasibility of Mindful Self-Care for Caregivers (MSCC) for parents of children with Autism Spectrum Disorders (ASD) (N=13). The intervention was offered as a co-located care model in a pediatric specialty center where the participants' children received care. Results demonstrated that the intervention was: highly acceptable to all stakeholders (i.e., participants, the group facilitator, and center administration) and could be conducted safely by a masters-level practitioner with minimal resources. Further, secondary measures support a hypothesized interventional model of MSCC, demonstrating gains in mindfulness skills and sense of competency in the parenting role reduced perceived stress and depression in parents of children with ASD.
\end{abstract}

Keywords Mindfulness-based cognitive behavior therapy $\cdot$ Mindful self-care $\cdot$ Caregivers $\cdot$ Autism spectrum disorders

\section{Introduction}

Parents and caregivers of children with ASD face multiple challenges which significantly increase parental stress, evident when "parenting demands exceed the expected and actual resources available to parents that permit them to succeed in the parent role" (Deater-Deckard et al., 2013), or defined by "a person's self-report of adaptive capacity overload" (Kupst et al., 2015). Such stressors may covary with challenging child behaviors, lack of access to effective treatments for their children, the financial burden of ASDrelated therapies, general lack of support or understanding by others (e.g., on public outings), and discord among parents and other family members related to parenting practices (Sharpley, 1997).

Katherine M. Bellone

Katherine.bellone@utsouthwestern.edu

1 Department of Psychiatry, University of Texas Southwestern Medical Center, 5323 Harry Hines Blvd, Mail Code 9086, Dallas, TX 75390, USA

2 Department of Population and Data Sciences, University of Texas Southwestern Medical Center, Dallas, TX, USA

3 Children's Health System of Texas, Dallas, TX, USA
Previous research on stress among parents of children with disabilities has found that overall, parents of children with disabilities experience clinically significant levels of stress compared to parents of typically-developing children (Hayes \& Watson, 2013). However, even beyond this, parents of children with ASD experience significantly more stress than parents of children with other disabilities, such as Down Syndrome or intellectual disability (Hayes \& Watson, 2013). Further, the percentage of parents endorsing negative perceptions of parenting is highest among parents of children with autism when compared to parents of children with special health care needs or emotional/behavioral concerns other than ASD (Schieve et al., 2007). Chronic parental stress not only reduces a caregiver's ability to sustain daily roles and increases potential for developing mental health concerns, but also can negatively impact child outcomes (Davis \& Carter, 2008). For example, research has suggested a bi-directional relationship between parental stress and behavior problems in children with ASD, such that over time, the presence of one factor tends to worsen the other (Lecavalier et al., 2006). Additionally, beyond worsening behavior problems, high levels of parental stress can reduce the positive treatment gains from early instructional interventions for children with ASD (Osborne et al., 2008).

Although chronic parental stress likely has a negative effect on parent outcomes, research also demonstrates that 
parental stress has direct and measurable effects on child outcomes for children with ASD. Supportive programming for parents of children with ASD has been found to result in improvements in the child's adaptive skills, communicative skills, and ASD-specific behaviors (Tonge et al., 2014). As additional evidence of this impact, Singh et al. (2006) demonstrated that training parents in mindful parenting approaches led to improvements in challenging behaviors, including noncompliance and self-injury, among children with ASD (2006). Thus, in addition to the common practice of teaching behavioral management strategies to parents, researchers note the importance and benefits of also teaching parents coping skills to help them with their own distress (Anderson, 2010). Clearly, helping parents develop better coping styles to manage chronic stress is beneficial to the family system as a whole.

Over the last decade, research in the area of child-focused intervention and parenting increasingly includes mindfulness-based strategies. One prolific line of research started with Bogels et al. (2008), who conducted a study utilizing mindfulness based cognitive-behavioral therapy programming (MBCT; Teasdale et al., 1995) for parents along with their children with diagnoses of Attention-Deficit/Hyperactivity Disorder, Oppositional Defiant Disorder/Conduct Disorder, and ASD. Results indicated positive improvements for parents and children and supported the benefits of specifically targeting "Mindful Parenting." Based on the observed benefits, Bogels and her collaborators have continued this line of investigation to emphasize the positive effects of offering parent and child mindfulness sessions in parallel (e.g., de Bruin et al., 2015; Ridderinkhof et al., 2018).

\section{Primary Purpose of the Study}

Given the efficacy of mindfulness-based stress reduction (MBSR; Kabat-Zinn, 1990, 2013) and MBCT (Teasdale et al., 1995) in reducing symptoms of depression and anxiety (Hofmann et al., 2010) as well as mental health concerns associated with chronic pain (Veehof et al., 2016), Lumpkin and Jarrett (2015) developed Mindful Self-Care for Caregivers (MSCC). Their practical manual was developed to guide seasoned mental health professionals in facilitating improved (mindful) self-care of groups of a) lay (not professional) caregivers and b) formal (professional) caregivers alike. The primary purpose of this proof of concept study was to evaluate the extent to which MSCC was feasible, safe and acceptable for parents of youth with ASD, according to both the parents and the MSCC facilitator.

We hypothesized that MSCC would benefit caregivers of individuals with ASD, such that the caregivers, like the facilitator, would find the intervention acceptable, feasible, and safe. Additionally, we sought to explore our hypothesis that MSCC would lower depression, anxiety and stress of parents. We proposed a model of behavior change whereby improvements in mindfulness, as well as increased self-efficacy in parenting or caregiving mediate the MSCC outcome of lowered depression, anxiety and stress (Jarrett, 2018). We speculate that once caregivers understand and experience the basics of mindfulness, they may learn how stress is generated and can begin to use mindfulness as an alternative response to stress.

\section{Method}

\section{Recruitment and Screening}

Sixteen parents were recruited by word of mouth (e.g., behavioral health provider mentioned during appointment), as well as flyers in common areas of a pediatric specialty clinic for individuals with developmental disabilities (e.g., waiting area, exam rooms). Any parent whose child had a diagnosis of ASD, who spoke English, and who received care at the clinic was eligible for participation. Of the 16 parents who expressed interest, 13 individuals signed the informed consent form and met the inclusion/exclusion criteria.

Following verbal and written informed consent, a psychiatrist conducted the Mini-International Neuropsychiatric Interview English version 7.0.2 (M.I.N.I) as a screening tool to aid in identifying whether interested caregivers might be at risk for an adverse psychological experience. The M.I.N.I. 7.0.2 is a brief, structured interview that aligns with diagnostic criteria from the Diagnostic and Statistical Manual of Mental Disorders- Fifth Edition (DSM-5; APA, 2013) to assess common psychiatric conditions (Sheehan, 1998, 2016). We screened for the following presenting psychiatric diagnoses, which in prior research, have been associated with a higher likelihood of a negative experience during mindful practice (e.g., excessive fear, feeling disconnected from self, recalling previous negative physical and/or psychological trauma; Kuijpers et al., 2007; Schlosser et al., 2019; Sharma, Mahapatra, \& Gupta, 2019), using specific sections of the M.I.N.I.: any psychotic disorder and PostTraumatic Stress Disorder. Given that mindfulness is not currently considered a first-line treatment for an active Alcohol Use Disorder or Substance Use Disorder, these conditions were also excluded. Screening required approximately 15-20 min per participant. Upon screening, we obtained one positive screen for a past history of alcohol use disorder; however, the participant no longer met criteria for current diagnosis.

Of the 13 eligible parents, one parent dropped out prior to the first session because she could not arrange childcare for one of her children. Therefore, the intention to treat sample consisted of 13 individuals with the following 
characteristics: 12 females, one male, of which 10 identified as White and 3 identified as Hispanic. Ten participants provided detailed demographic data after the study was completed related to their and their child's background. The mean participant age was 43.1 years (range $35-56$ years), with children's ages ranging from 7 to 18 years (average age of child was 10.9 years). Two participants identified receiving additional external support/intervention during the study (one parent received individual therapy and one received both therapy and pharmacologic intervention). Of the participants who provided detailed background information, the majority (90\%) had little to no experience with mindfulnessbased practice prior to the study. In terms of educational backgrounds, the majority of participants reported having some level of post-secondary education, ranging from "some college" to holding a professional degree. In order to capture the functioning or severity of ASD symptoms experienced by a participant's child, parents were asked to identify the level of support their child requires using descriptions that correspond to the Autism Severity Levels from the DSM-5 (APA, 2013). Based on provided responses, most parents perceived their children as requiring "some support" (70\%) and the other $30 \%$ as requiring "a lot of support," mapping on to Severity Levels 1 and 2. Eleven of the 13 caregivers who participated in this study also participated in outpatient behavioral therapy services which specifically targeted child behaviors.

\section{Mindful Self-Care for Caregivers (MSCC)}

Mindful Self-Care for Caregivers (MSCC) is an 8 week series of eight, $1.5 \mathrm{~h}$ classes plus routine suggested practices to complete at home. Like Mindfulness-Based Stress Reduction (MBSR) and Mindfulness Based Cognitive Therapy (MBCT), MSCC combines graduated training in mindful, self-care skills, as well as cognitive therapy's decentering and diffusion toward the usual stream of automatic cognitions, including those related to stressors associated with caregiving. As stated, MSCC is designed to be delivered across an 8 week protocol, making the time investment for MSCC comparable to that of MBSR and MBCT. Modifications to MBSR and MBCT included the focus on caregiver stress.
Classes were structured to gradually move from simple to more challenging levels of formal and informal mindfulness practices. A session typically included space for open expression/sharing, use of a reflective reading for discussion, and a guided meditative practice. Guided practices in the initial few sessions included brief breathing exercises with high levels of supportive guidance through facilitator verbalizations and gradually transitioned to longer exercises (e.g., 20 min meditation) with less facilitator verbalization. Conceptually, the discussion and reflection were intended to help caregivers understand how stress is generated by automatic ways of avoidant coping and how to use mindful acceptance as an alternative stress response. Participants learn to see stress and distress associated with caregiving, not as a condition to be treated, but instead as a natural, although sometimes painful, state to be experienced. This alters one's relationship to the stress, feelings of loss, and associated grieving that accompany parenting a child with special needs so that depression and anxiety no longer "complicate" the parenting relationship. The words "Self-Care" were added to the title to emphasize the importance of the caregiver acknowledging and responding to their own needs.

Table 1 below lists the focus of each of the eight sessions of MSCC.

We provided MSCC in a group format at the pediatric specialty center where the participants' children received care. Three cohorts of participants completed the intervention. For the first cohort, scheduling occurred at the same time as a child-focused social skills group to increase convenience of attending. The subsequent two groups were scheduled in the late morning on a weekday to accommodate participants' schedules while their children attended school.

The MSCC manual is constructed to make mindfulness group facilitation accessible to providers with basic skills in providing mental health interventions. The MSCC group was facilitated by a Licensed Professional Counselor, who prior to the initial study-related training (described below), had no formal mindfulness training, but participated in informal personal practice through yoga. Initial orientation and training for facilitating included $6 \mathrm{~h}$ of group practices for MSCC (over three $2 \mathrm{~h}$ sessions) and was conducted by two licensed psychologists and a psychiatrist. Training consisted of reviewing, discussing, and modeling implementation of

Table 1 Session content for mindful self-care for caregivers (MSCC)

\begin{tabular}{llll}
\hline Session & Title and focus & Session & Title and focus \\
\hline 1 & Awareness and automatic pilot & 5 & $\begin{array}{c}\text { "Allowing/letting be" (as the principle } \\
\text { of acceptance) } \\
\text { Mindful responses to others }\end{array}$ \\
2 & Living in our stories (vs. our immediate experience) & 6 & Mindful self-care \\
3 & The pleasure and power of being present & 7 & Maintaining and extending new learning \\
\hline
\end{tabular}


all materials from the MSCC manual. Trainers also provided specific feedback on successful group facilitation practices, such as adjustments that can be offered for particular practices (e.g., allowing participants to practice the body scan exercise in various positions based on preference: seated, standing, lying down).

In preparation for potential adverse events, the primary investigators developed a safety protocol which specified when and how to assess risk and support safety of the participants. Additional considerations included how/when to engage safety officers, arranging childcare for the child of any participant in distress, and handling urgent communication received outside of a session. Triggers for activating the safety protocol included verbalizations during group practice related to intent to inflict harm on self or others, self-reported distress related to the practice (e.g., recalling a previous trauma), or any severe behavioral reaction that was considered to be unusual or unexpected for a particular participant. All study procedures were reviewed, approved, and monitored by the university Institutional Review Board.

\section{Measurement}

The primary goals of this study were to develop and test the safety, tolerability, acceptability, and feasibility of MSCC for parents of youth with ASD. Secondary aims involved a preliminary test of a hypothesized model of change whereby increased skill in mindfulness and/or increased self-efficacy in parenting or caregiving may mediate decreased stress, anxiety and depression.

\section{Primary Outcomes}

\section{Safety}

We defined a safety concern as an adverse event, which consisted of any study-related event that was unanticipated and/ or posed possible risk of harm, resulted in distress to a participant/study team member, or compromised confidentiality.

\section{Tolerability and Acceptability}

Participants, staff, and facilitators provided qualitative and quantitative feedback on the composite package (a) MSCC: the overall experience of MSCC and its components including exercises, handouts, and group practice and (b) research methods including the assessment battery and the protocol. Raters used six Likert-type scales to provide quantitative data, in addition to open-ended questions to capture qualitative comments on areas that needed improvement. The six Likert scales measured participant experiences with MSCC for the following components: comfort with exercises (e.g., breathing practice), value of handouts, overall participant enjoyment, completing study-related questionnaires, importance of materials, and overall learning. The ratings on the Likert scales captured responses in terms of the strength of the participant's experience/agreement with each component, ranging from $1=$ not at all, $2=\mathrm{minimal} / \mathrm{a}$ little bit, $3=$ moderate/some, $4=$ highly/a high amount, $5=$ extremely $/$ the most possible. As a sample, one question read "How comfortable were you participating in exercises in group?" and the related choices were "Not Comfortable at All, Minimally Comfortable, Moderately Comfortable, Highly Comfortable, and Extremely Comfortable." We examined the means and standard deviations of these Likert scales to examine any changes reported from Week 1 to Week 8 of participation.

\section{Feasibility}

To demonstrate feasibility, we piloted and evaluated the described tools and research design in the participant sample paying particular attention to uptake, attrition, and missing data.

\section{Secondary Outcomes}

In order to examine relationships between participation in MSCC and mental health outcomes, various paper and pencil questionnaires were administered to participants prior to the first session, at the mid-point in the series (Week 4) and at the final session (Week 8). The measures that were used are described below and were chosen to look at relationships between parenting, stress management, parental mental health, and mindfulness as an interventional process.

\section{Stress}

Participant stress was measured using the National Institute of Health (NIH) Toolbox Perceived Stress Fixed Form Age $18+$ v2.0 (Salsman et al., 2013). This measure is part of the NIH Toolbox $®$, which is a collection of neuro-behavioral measures that quickly assesses a range of cognitive, emotional, and physical functions. The group of measures contained in the NIH Toolbox® ${ }^{\circledR}$ was normed on a large sample of people aged 3-85 years who were representative of the United States population based on gender, race/ethnicity, and socioeconomic status, with specific comparison groups identified for each measure (Gershon et al., 2013). The version of the Perceived Stress Fixed Form that was used in the current project is designed for individuals over the age of 18 and includes 10-items with a 5-point Likert-type response scale. Possible responses range from "Never to Very Often" and are designed to capture participants' perceptions of personal stress in the past month. While we considered measuring stress related to parenting specifically, a global measure of 
overall distress was selected given challenges with parenting stress tools for this particular purpose (i.e., measures with a ceiling age for the child lower than our clinic population, measures that are not specifically normed for caregivers of individuals with ASD and thus, it is unclear whether there is adequate sensitive to capture change). The Perceived Stress measure was ultimately chosen due to easy accessibility (e.g., available online at no cost) and strong psychometric properties, as well as its wide application.

\section{Depression}

To obtain an estimate of current depressive symptoms, the Patient Health Questionnaire (PHQ-9) was used. The PHQ-9 is a diagnostic tool used by health care providers to quickly and easily assess mental health disorders. It is a brief self-report measure that includes 9 items which assess symptoms of depression based on the criteria for diagnosis of a depressive disorder from the Diagnostic and Statistical Manual of Mental Disorders, Fourth edition. The PHQ-9 uses a 4-point Likert-type scale which ranges from 0 (not at all) to 3 (nearly every day) and asks participants to endorse their rating based on experiences over the last 2 weeks. The measure has previously been determined to have acceptable reliability, validity, and specificity as an assessment tool for symptoms of depression in adults with similar demographics (Kroenke et al., 2001).

\section{Self-Efficacy in Parenting}

To assess participants' perceptions of their efficacy and competence as parents, the Parenting Sense of Competence Scale (PSOC; Johnston \& Mash, 1989) was selected. This widely-used measure contains 16 items that specifically assess parental competence along two dimensions, Satisfaction and Efficacy using a 6-point Likert-type scale. Response options range from "Strongly Disagree to Strongly Agree." The PSOC has been determined to demonstrate acceptable reliability and validity, in addition to undergoing updated norming (Gilmore \& Cuskelly, 2008). The PSOC has been used in previous research with parents of individuals with ASD (e.g., Iadarola et al., 2018; Mathew et al., 2019).

\section{Mindfulness}

In order to measure the degree to which participants felt they experience daily events in a mindful manner, the Five Facet Mindfulness Questionnaire-15 (FFMQ-15; Baer et al., 2012) was used. The FFMQ-15 is a shortened form of the FFMQ, which contains 39-items. Both tools assess the same five facets of inner experience, including Observing, Describing, Acting with Awareness, Non-Judging and Non-Reactivity. Participants rate their daily inner experiences across a 5-point Likert-type scale, with responses that range from "Never or very rarely true to Very often or always true." Gu et al. (2016) evaluated the factor structure and psychometric properties of the FFMQ-15, finding adequate reliability, convergent validity, and sensitivity to change over time (such as is commonly used to assess change as a result of participation in mindfulness-based interventions) in a similar adult sample.

\section{Research Design/Statistical Analyses}

The analysis was based on an intention to treat sample (i.e., all consenting, enrolled participants). The research design was an open trial to assess safety, feasibility, and acceptability in an intention to treat sample of 13 parents of youth with ASD. All secondary measures were collected pre (at consent-Week 0 and 1), mid (at Week 4) and post treatment (at Week 8), while evaluative measures of acceptability were gathered at the midpoint (Week 4) and final point (Week 8) to allow for some level of experience prior to soliciting feedback. When a participant missed a session that included administration of measures, the investigators made efforts to have the measures completed by sending the measures electronically when possible or asking participants to complete them upon their arrival for the next session prior to that session commencing. Data were entered into REDcap (Harris et al., 2009). A quantitative psychologist provided descriptive statistics on the sample outcomes using Statistical Analysis Software (SAS) and participated in interpretation of data to inform advisability of future studies/trials. Proc Mixed in SAS was chosen, as it is tolerant of missing data and is considered very robust when data are missing at random (Mallinckrodt et al., 2004).

\section{Results}

\section{Safety}

As defined above, no adverse events occurred during the implementation of this project. The safety protocol did not need to be activated during or after the project. In fact, there have not been any reported adverse events during a session to date (1 year after formal data collection ended, but with continued weekly sessions being held).

\section{Feasibility}

To demonstrate feasibility, we developed and piloted the described tools in the participant sample. Twelve of the 13 participants in the sample attended at least one session (one participant dropped out prior to session 1 due to inability to find childcare for her child's younger sibling). Participants 
attended an average of 5 of the 8 sessions, with a median and mode of 7 out of 8 sessions. Further, the masters-prepared mental health provider was able to facilitate the intervention effectively with an initial $6 \mathrm{~h}$ of face to face training in using the treatment manual to promote mindful practices for caregivers (Lumpkin \& Jarrett, 2015).

With respect to the feasibility of the research design and data collection, 3 of the 12 participants completed baseline measurements only, one of which had missing observations for two of the efficacy measures on the PSOC resulting in missing data for the Total Score and the Efficacy Score. The remaining 9 participants were included in the longitudinal analyses; one of the 9 cases was missing all data collected at Week 4 and another case was missing all data collected at Week 8.

\section{Tolerability and Acceptability}

Participants completed Likert scales to rate their experiences in terms of comfort with exercises (e.g., breathing practice), perceived value of handouts, overall enjoyment, completion of study-related questionnaires as part of the protocol, perceived importance of materials, and overall learning. The majority (85.7-100\%) of participants rated all aspects of intervention and research participation as "highly or extremely" acceptable or positive (reflected by either a 4 or 5 on the 5-point Likert scale) both at Week 4 and at Week 8 (See Figs. 1 and 2). "Top box" ratings, meaning best or most acceptable and reflected by either a rating of 4 or 5 , were provided by $92.9 \%$ of participants in Week 4 and $97.6 \%$ of participants in Week 8 (See Fig. 1). As demonstrated in Fig. 2, among the ratings, improvements were noted between Week 4 and Week 8 in terms of ratings of comfort with the exercises, enjoyment of the practices, usefulness of the handouts, and overall learning. Regarding completion of study-related questionnaires as part of the research protocol, several raters provided improved ratings between Week 4 and Week 8, but one rater changed from "highly to moderately" satisfied with the questionnaires. Taken together, these data demonstrate that participants found the intervention highly acceptable and for some participants, acceptability of the intervention improved over time.

When asked for qualitative feedback, several written comments from the participants included: "love it!;" "group participation is great," "group has been helpful even when having a bad day;" and "I found the sessions to be rewarding and helpful. My daily stress has been reduced and my ability to handle stressful parenting moments has improved." Participants were asked to provide ideas for improvement and several participants wrote "to continue sessions" (past Week 8). The only negative written feedback that was received was related to logistics of attendance, such as parking availability and time of the group coinciding with rush hour.

Qualitative feedback from the facilitator was very positive in terms of ease of implementation based on the guides in the MSCC manual and positive personal experiences during interactions with participants. Additionally, several staff members in the center provided unsolicited positive feedback related to perceived changes in the participants (e.g., observed during check in/out), and further, medical clinic staff asked for the option to participate in their own MSCC sessions as formal/professional caregivers of individuals with ASD. Perhaps the greatest demonstration of acceptability is that participants requested to continue to attend MSCC sessions after the research protocol ended. Therefore, the investigators approached clinical leadership and successfully obtained administrative support to offer the program
Fig. 1 Percentage of participants providing highest acceptability ratings for all components of MSCC across weeks 4 and 8 . Total percent of participants providing a rating of 4 or 5 (i.e., top box) in response to the acceptability of aspects of 8 weeks of Mindful Self-Care for Caregivers (MSCC), where $4=$ Highly agree and $5=$ Extremely agree with item (See text for items) or high acceptability

\section{Percentage of Total Top Box Ratings}

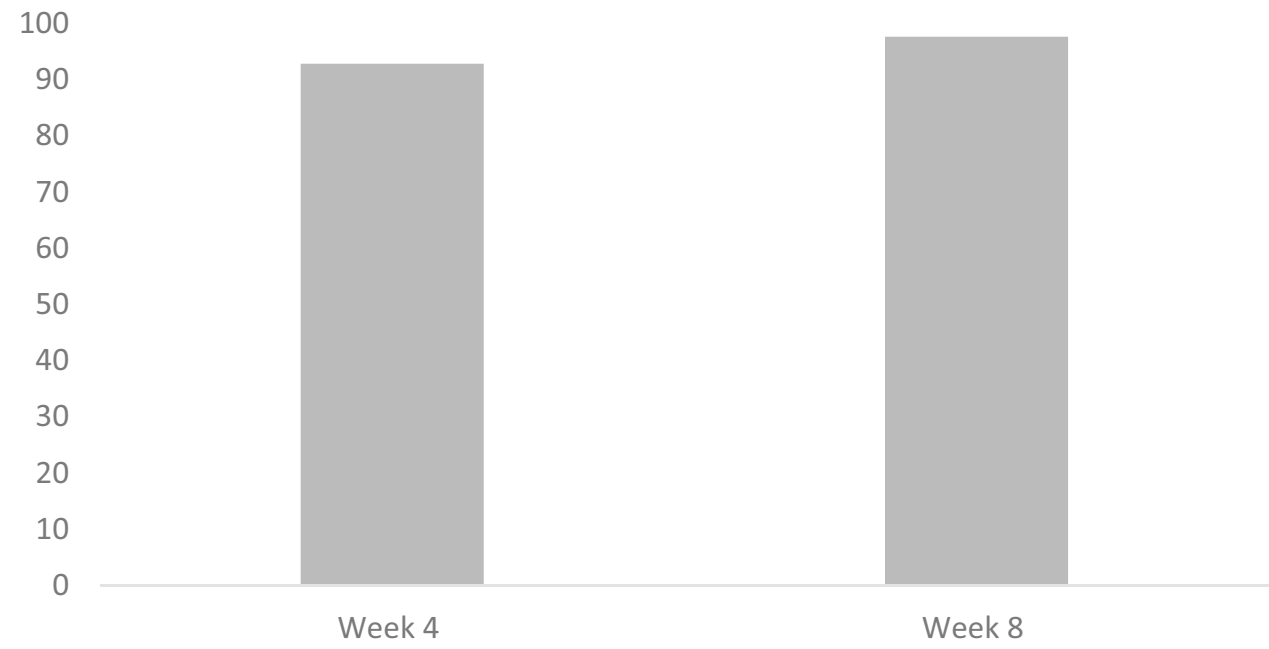


Fig. 2 Participant ratings of acceptability of Mindful SelfCare for Caregivers (MSCC): overall and components. Percentage of participants, across weeks 4 and 8 , rating the acceptability of components of MSCC on a 5-point Likert scale where Low acceptability is reflected by a 1 or 2, Moderate acceptability is reflected by a 3 and High acceptability is reflected by a 4 or 5

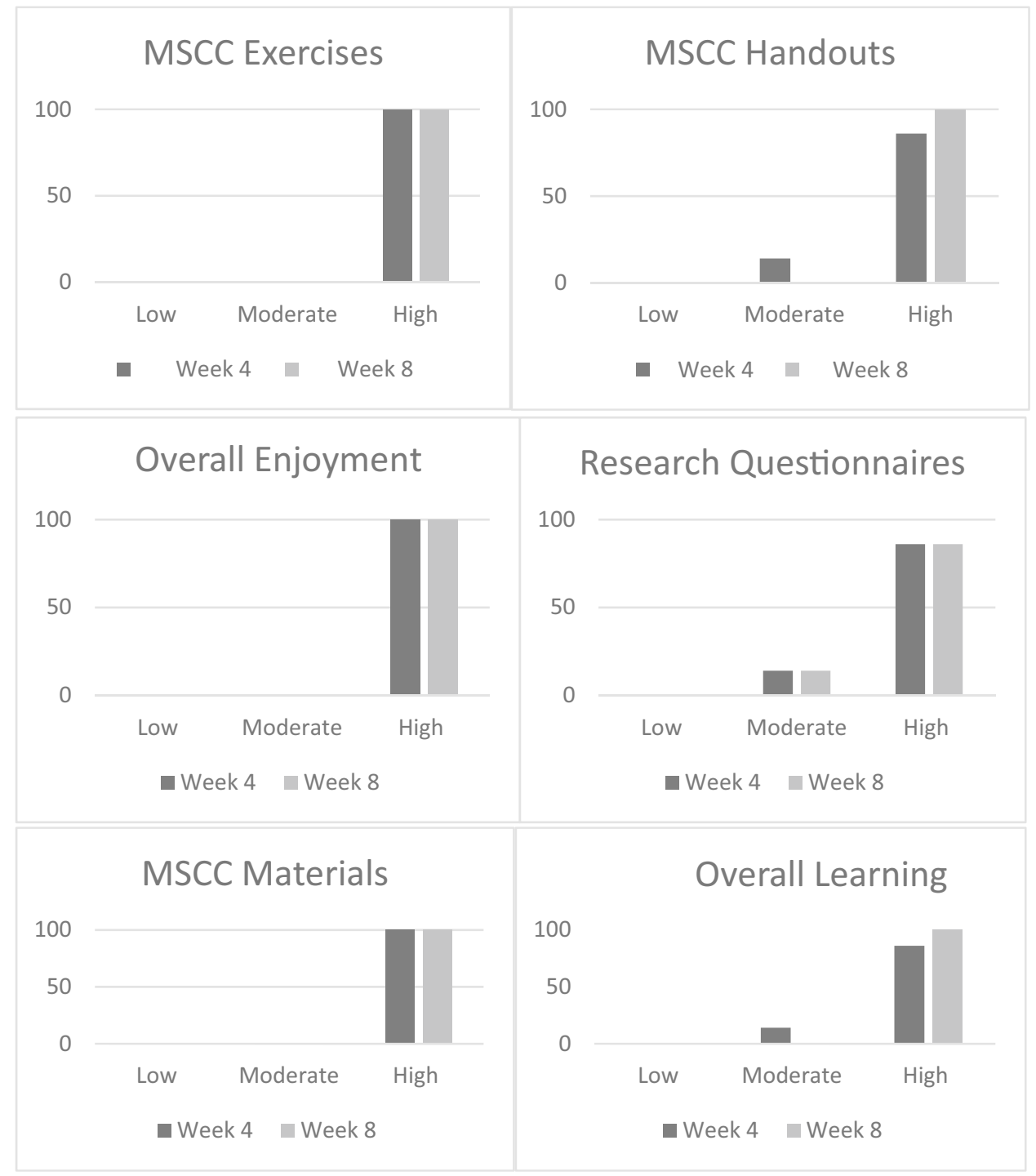

indefinitely. At the time of this writing, the group continues to be offered weekly and is well attended.

Related to acceptability and feasibility of implementation, following completion of the formal protocol and extension as a clinical offering, an unintended second phase of implementation occurred due to social distancing and infection control recommendations related to COVID-19. Beginning in the spring of 2020, hospital guidelines were developed based on the recommendations of the Centers for Disease Control and Prevention, which required suspension of gatherings larger than 5 people. This directly impacted the ability to safely hold the weekly parent group at the clinic. The group facilitator developed a virtual group using an encrypted and HIPAA-compliant audio-video platform and continued to offer weekly sessions remotely. Participants provided informed consent allowing them to attend in this manner by signing into the web address from their homes. This allowed the group to be extended to participants who previously had difficulty attending the physical group due to logistical barriers (e.g., transportation, childcare). At the writing of this paper, on average, 10 parents attend regularly via the virtual platform.

\section{Secondary Outcomes}

Beyond finding the intervention feasible, safe, and acceptable, we hypothesized that participation in MSCC would lower depression, anxiety and stress of participants by increasing their skill in practicing mindfulness, as well as self-efficacy/positive beliefs about themselves as a parent or caregiver. In order to measure parental effects, we asked parents to complete paper and pencil measures at Week 1, Week 4 (mid-point) and Week 8 (final session). Measures that were included assessed parental stress, symptoms of depression, sense of competence as a parent, and degree to which the parent felt he/she had acquired mindfulness skills. 
The following two conceptual models show the hypothesized relationships among parenting children with ASD, stress management, and parental mental health (Model A; [Jarrett, 2018]) and the putative mechanisms through which MSCC may improve outcomes (Model B; [Jarrett, 2018]).

A. Model of emotional states within parenting a child with ASD (Jarrett, 2018)

\section{Depression}

Clinically significant reductions in self-reported depressive symptoms were found. Overall, participants experienced a $39.5 \%$ reduction in reported symptoms of depression on average from Week 1 to Week 8 , representing a reduction from being classified in the "moderate depression" range to the low end of the "mild depression" category. This resulted

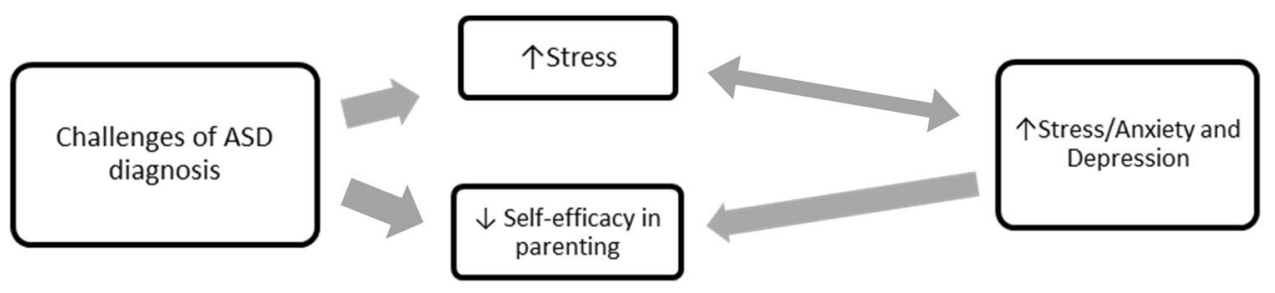

B. Interventional Model/Mindful Self-Care for Caregivers

(Jarrett, 2018)

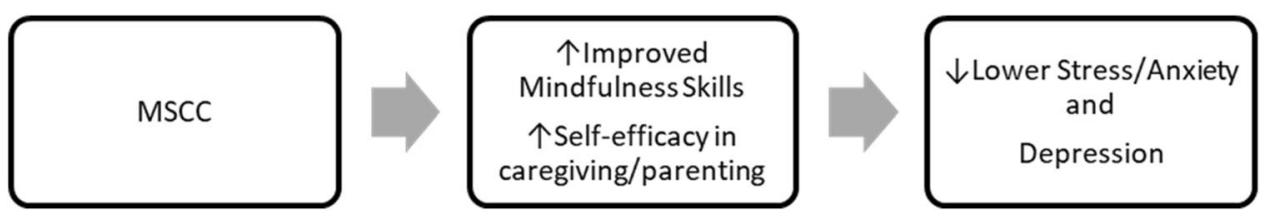

A repeated measures, analysis of variance (ANOVA) was used to analyze the continuous measures with time $(1,4$, and 8 weeks) as the repeated measure. For those measures with a $p$ value less than 0.05 , suggesting that the changes were unlikely to be due to chance, a Bonferroni post hoc pairwise comparisons test was conducted. These calculations are provided in Table 2. For all measures, a Cohen's d was calculated to obtain an estimate of effect size. When interpreting these effect size estimates, 0.2 is considered a "Small" effect, 0.5 is considered a "Medium" effect and 0.8 is considered a "Large" effect. Effect size estimates are provided in Table 3.

\section{Stress}

Clinically significant reductions in self-reported stress were found. Overall, participants experienced a $15.1 \%$ decrease in perceived stress on average from Week 1 to Week 8. This resulted in a Cohen's d effect size estimate of 0.6 (Medium effect) and is consistent with the Interventional Model of MSCC in this initial sample. in a Cohen's d effect size estimate of 0.65 (Medium effect) and is consistent with the Interventional Model of MSCC in our initial sample.

\section{Parental Sense of Competence}

Findings indicate statistically significant improvements from Week 1 to Week 8 in overall parental sense of competence (average rating of 64.44 at Week 1 and 75.87 at Week 8), parental efficacy (average rating of 26.22 at Week 1 and 30.85 at Week 8), and parental satisfaction (38.22 at Week 1 to 44.86 at Week 8). These findings result in effect size estimates ranging from 0.997 to 1.42 (all Large effects) and is consistent with the Interventional Model of MSCC for the initial sample.

\section{Mindfulness}

A statistically significant change in self-rated mindfulness from Week 1 to Week 8 (average rating of 50 at Week 1 and 56.02 at Week 8) was obtained for the total score, along with 
Table 2 Secondary outcomes using repeated measures ANOVA at Weeks 1, 4, and 8 of MSCC

\begin{tabular}{|c|c|c|c|c|c|c|}
\hline \multirow[t]{2}{*}{ Measure } & \multirow[t]{2}{*}{ Visit week } & \multirow[t]{2}{*}{ Mean score } & \multicolumn{2}{|c|}{ Model } & \multicolumn{2}{|c|}{ Pairwise post hoc tests } \\
\hline & & & $\mathrm{F}_{2,14}$ & $\mathrm{p}$ value & Pairs (week) & $\begin{array}{l}\text { Bonferroni } \\
\text { adjusted p } \\
\text { value }\end{array}$ \\
\hline \multirow[t]{3}{*}{ Parenting sense of competence total score } & 1 & 64.44 & 9.14 & 0.0029 & 1 vs 4 & 0.3848 \\
\hline & 4 & 68.78 & & & 1 vs 8 & 0.0024 \\
\hline & 8 & 75.87 & & & 4 vs 8 & 0.0719 \\
\hline \multirow[t]{3}{*}{ Satisfaction } & 1 & 38.22 & 4.58 & 0.0295 & 1 vs 4 & $>0.9999$ \\
\hline & 4 & 40.31 & & & 1 vs 8 & 0.0292 \\
\hline & 8 & 44.86 & & & 4 vs 8 & 0.2091 \\
\hline \multirow[t]{3}{*}{ Efficacy } & 1 & 26.22 & 5.82 & 0.0145 & 1 vs 4 & 0.3486 \\
\hline & 4 & 28.50 & & & 1 vs 8 & 0.0127 \\
\hline & 8 & 30.85 & & & 4 vs 8 & 0.3552 \\
\hline \multirow[t]{3}{*}{ Patient health questionnaire-9 total score } & 1 & 11.00 & 2.65 & 0.1056 & 1 vs 4 & \\
\hline & 4 & 6.57 & & & 1 vs 8 & \\
\hline & 8 & 6.66 & & & 4 vs 8 & \\
\hline \multirow[t]{3}{*}{ Five facet mindfulness questionnaire-15 total score } & 1 & 50.00 & 4.81 & 0.0257 & 1 vs 4 & $>0.9999$ \\
\hline & 4 & 51.87 & & & 1 vs 8 & 0.0253 \\
\hline & 8 & 56.02 & & & 4 vs 8 & 0.1884 \\
\hline \multirow[t]{3}{*}{ Observing } & 1 & 10.00 & 11.65 & 0.0011 & 1 vs 4 & 0.2396 \\
\hline & 4 & 11.03 & & & 1 vs 8 & 0.0008 \\
\hline & 8 & 12.64 & & & 4 vs 8 & 0.0426 \\
\hline \multirow[t]{3}{*}{ Describing } & 1 & 10.33 & 4.1 & 0.0397 & 1 vs 4 & $>0.9999$ \\
\hline & 4 & 10.73 & & & 1 vs 8 & 0.0439 \\
\hline & 8 & 11.99 & & & 4 vs 8 & 0.1884 \\
\hline \multirow[t]{3}{*}{ Acting } & 1 & 9.11 & 0.06 & 0.9450 & 1 vs 4 & \\
\hline & 4 & 9.29 & & & 1 vs 8 & \\
\hline & 8 & 9.28 & & & 4 vs 8 & \\
\hline \multirow[t]{3}{*}{ Non-judging } & 1 & 11.00 & 0.21 & 0.8154 & 1 vs 4 & \\
\hline & 4 & 11.42 & & & 1 vs 8 & \\
\hline & 8 & 11.36 & & & 4 vs 8 & \\
\hline \multirow[t]{3}{*}{ Non-reactivity } & 1 & 9.56 & 2.77 & 0.0970 & 1 vs 4 & \\
\hline & 4 & 9.47 & & & 1 vs 8 & \\
\hline & 8 & 10.67 & & & 4 vs 8 & \\
\hline \multirow[t]{3}{*}{ Perceived stress total score } & 1 & 21.56 & 2.02 & 0.1692 & 1 vs 4 & \\
\hline & 4 & 18.69 & & & 1 vs 8 & \\
\hline & 8 & 18.31 & & & 4 vs 8 & \\
\hline \multirow[t]{3}{*}{ Perceived stress unstandardized T-score } & 1 & 45.84 & 2 & 0.1722 & 1 vs 4 & \\
\hline & 4 & 41.38 & & & 1 vs 8 & \\
\hline & 8 & 39.97 & & & 4 vs 8 & \\
\hline
\end{tabular}

the Observing and Describing subscales. This represents a Cohen's d effect size estimate of 1.020 (Large effect) and is consistent with the Interventional Model of MSCC in this initial sample.

While the sample size was too small to test the model fully, the effect sizes that were obtained demonstrate adequate power. Based on these findings, we have preliminary evidence to support the MSCC interventional model of behavior change, evidenced by clear statistically and clinically significant improvements in mindfulness and beliefs about competency, efficacy, and satisfaction as a parent, with clinical improvements in stress, depression, and anxiety observed. 
Table 3 Effect size for secondary outcome after MSCC

\begin{tabular}{|c|c|c|c|c|c|}
\hline \multirow[t]{2}{*}{ Variable } & \multicolumn{2}{|l|}{ Mean } & \multicolumn{2}{|c|}{ Contrast week 1 vs week 8} & \multirow{2}{*}{$\begin{array}{l}\text { Interpretation } \\
\text { of effect size }\end{array}$} \\
\hline & Week 1 & Week 8 & Mean difference & Cohen's d & \\
\hline Parenting sense of competence scale total score & 64.44 & 75.87 & -11.43 & -1.420 & Large \\
\hline Satisfaction & 38.22 & 44.86 & -6.64 & -0.997 & Large \\
\hline Efficacy & 26.22 & 30.85 & -4.63 & -1.137 & Large \\
\hline Patient health questionnaire-9 total score & 11.00 & 6.66 & 4.34 & 0.650 & Medium \\
\hline Five facet mindfulness questionnaire-15 total score & 50.00 & 56.02 & -6.02 & -1.020 & Large \\
\hline Observing & 10.00 & 12.64 & -2.64 & -1.603 & Large \\
\hline Describing & 10.33 & 11.99 & -1.65 & -0.927 & Large \\
\hline Acting & 9.11 & 9.28 & -0.17 & -0.093 & Small \\
\hline Non-judging & 11.00 & 11.36 & -0.36 & -0.167 & Small \\
\hline Non-reactivity & 9.56 & 10.67 & -1.11 & -0.673 & Medium \\
\hline Perceived stress fixed form total score & 21.56 & 18.31 & 3.25 & 0.607 & Medium \\
\hline Perceived stress unstandardized T-score & 45.84 & 39.97 & 5.87 & 0.630 & Medium \\
\hline
\end{tabular}

\section{Discussion}

This study evaluated the acceptability, feasibility, and safety of Mindful Self-Care for Caregivers (MSCC) from the perspective of the parents of children with ASD and also from the facilitator of MSCC. Results showed that the relevant stakeholders (i.e., participants, the group facilitator, and the center staff) found the intervention acceptable, feasible and safe and valued MSCC enough to ask to continue it after the research protocol ended and through online delivery during the COVID-19 pandemic. Considerations that likely contributed to the feasibility and safety of the intervention include clear and thorough screening protocols, as well as availability and review of a safety protocol to improve facilitator comfort. Although no participants were excluded through this screening method, conducting the M.I.N.I. likely resulted in a pool of participants who were at low risk for an adverse reaction to this mode of interventional practice. Additionally, with proper screening practices in place, findings suggest that MSCC (Lumpkin \& Jarrett, 2015) can be implemented safely by mental health clinicians who have minimal formal mindfulness training.

Further, in terms of feasibility and acceptability, three points are noteworthy. First participants asked to continue MSCC post-protocol, and the health system and facilitator agreed to do so. Second, the intervention demonstrated ecological validity through its fast and effective adaptation to a web-based platform during the COVID-19 pandemic. Given the widespread mental health burden associated with the global pandemic (National Alliance on Mental Illness [NAMI], 2020), the ability to offer meaningful interventions to improve overall mental wellness in a way that is accessible, safe, and easy is paramount. Although unplanned, we were able to demonstrate that MSCC successfully bridged this need and continues to benefit participants in this manner. Third, in terms of feasibility, we demonstrated positive results following a relatively limited treatment intervention consisting of 8 weeks of practice, as compared to the 12-16 week protocols included in many similar interventions. With ongoing real-world challenges of funding and insurance coverage for mental and behavioral health treatments, as well as the ever-present issue of patient retention and attrition, it is increasingly important to consider interventions that can be effectively utilized in a short duration as one way to maximize benefits to participants. In considering all of these points together, MSCC demonstrated sustainability and scalability as an intervention that was able to be effectively implemented over time with limited resources, as well as easily adjusted to meet the unplanned needs of an unprecedented situation (i.e., shelter in place initiatives), to a participant group that is historically underserved (i.e., caregivers of children with ASD).

Based on clinical experiences and the growing body of literature on mindfulness-based practices, we hypothesized that MSCC would reduce parental stress, anxiety, and depression in caregivers of children with ASD by improved mindfulness skills and self-efficacy in caregiving and parenting. Although our sample size was small, the effect sizes demonstrate that the sample was adequately powered to allow preliminary supportive evidence of the Interventional Model of MSCC for caregivers of individuals with ASD (Jarrett, 2018). High to moderate effect sizes suggested that caregivers, in this case, parents, benefitted from participating in terms of gains in perceived self-efficacy, competence and satisfaction as a parent, as well as gains in mindfulness skills. Through their participation in MSCC, parents, on average, also experienced clinically significant reductions in stress and depression. This finding is important not only for personal well-being, but acknowledged connections between parental mental health and child mental health. As all of the 
children of the participants received care at the center, this allowed informal observations of the downstream effects of parental participation in MSCC. Anecdotally, on numerous occasions, parents who had participated in the group verbalized improved resilience and cognitive flexibility when handling their child's challenging behaviors (e.g., "meltdowns", aggressive episodes, obsessive behaviors) which are associated with the diagnosis of ASD and known to negatively impact parental mental health (Sharpley et al., 1997).

\section{Limitations}

Despite the promising findings it is important to acknowledge limitations. First, the lack of a control group as well as the small sample size, along with its characteristics, limits both the generalizability of the findings, and the interpretative power of the underlying model. Even with robust recruitment efforts (e.g., flyers posted throughout the center, word-of-mouth from individual providers), the final sample was small and rather homogenous. In part, this finding underlies the legitimate challenges faced by caregivers of children with ASD in accessing support services. Future research should continue to examine potential barriers to participation in mental health interventions for caregivers of children with ASD to develop practice guidelines and delivery methods that may mitigate those barriers when possible, such as web-based platforms. For the purpose of this study, we sought to provide a group experience unique to caregivers of individuals with ASD; however, future studies could seek to potentially improve recruitment through inclusion of parents and caregivers of individuals with other chronic or developmental conditions, as much of the content would still be applicable, and likely, beneficial.

Related to the sample size, no males (fathers) successfully completed the intervention. Unfortunately, the male participant who started the intervention suffered a loss of employment in Week 2 and was unable to continue attending through Week 8. Importantly, as a sign of the level of personal meaning experienced and acceptability of the intervention, this participant remained in contact with the facilitator and subsequently joined some of the virtual group meetings, albeit outside of the scope of his research involvement. As participation in outpatient mental health interventions is historically lower for males than females, it will be important for interventions to continue to reflect on potential aspects of intervention design and implementation that may result in differences in attendance according to gender (World Health Organization, 2002). Evaluating MSCC with a diverse, large sample and adequate controls will be important.

We also acknowledge that we do not have fidelity information on facilitator behavior or on homework completion for participants. We relied on experiential feedback from the facilitator and participants to determine weekly "success" and adjust as needed. Anecdotally, weekly homework was an area that required adjustment and modeling of self-compassion by the facilitator due to parental worry related to failure to complete homework assignments. The emphasis for homework assignments became identifying feasible ways, even if very limited, to incorporate at least one meaningful mindfulness practice throughout the week, as participants had difficulty considering adding another "task" into their already hectic daily lives. For example, the facilitator helped participants brainstorm existing daily activities during which they could tune in to their breath and sensory experiences, such as washing dishes or taking a shower. Future studies could include use of fidelity checks and collection of homepractice logs for more objective behavioral measures.

\section{Implications and Future Directions}

Completing this proof of concept study suggested that MSCC was associated with clinically and personally meaningful changes for participants and that it was feasible, safe, and acceptable to stakeholders. Therefore, although our uncontrolled sample was small, we were able to begin to evaluate the underlying model of mental health challenges in parents of children with ASD and possible pathways to improvement. As is commonly cited in many group-based interventions, we cannot rule out the possibility that factors inherent to the group milieu (e.g., supportive interactions, connecting with individuals with similar experiences) contributed to the reductions in stress and depression. However, it should be considered that improvements in parental sense of competence, parental satisfaction, and parental sense of efficacy, in addition to mindfulness skill, would not be expected to shift through interpersonal engagement alone. Therefore, the information gleaned from this project can be used to help inform the advisability and design of a randomized controlled trial of MSCC designed to reduce stress, anxiety, and depression in parents of youth with ASD. Such a randomized controlled trial would inform clinical practice with the potential to improve future service provision to families of children with ASD and related disabilities.

One of the strengths of this proof of concept study was the practical approach of offering co-located services, such that parents of children with ASD were able to participate in an intervention that was specifically intended to improve their mental health within the same clinic where their children received medical, behavioral, and psychological care. Although the integration of mental health care within primary care settings has received increasing attention in recent years, providing support services for parents within pediatric practices has not received equal attention as a feasible way to reduce barriers to functioning and improve familycentered care to maximize outcomes. Leslie et al. (2016) reviewed family-focused preventive interventions that had 
been integrated into primary care settings. Overwhelmingly, the identified programs focused on behavioral management or parent training, which is undeniably important, but not sufficient when supporting family systems. As discussed by Pordes et al. (2018), for children with medical complexity (CMC), current care models often poorly integrate services and result in inadequate support for caregivers. The authors state, "An ideal care delivery system for CMC would partner with families to ease their financial, time, and emotional burden and provide caregiver support systems, such as increased in-home care, respite facilities, financial resources, and access to high-quality adult mental and physical health care" (Pordes et al., 2018).

Further, practice guidance from the Center for the Study of Social Policy (CSSP; Doyle et al., 2019) named "nurturing parents' competence and confidence" through intentional intervention as one of the three key practices used in pediatric interventions to support the social-emotional development of children and the parent-child relationship. Within these guidelines, the authors offer suggestions of common practices that can be implemented to achieve this aim, such as creating opportunities for parents to connect to other parents and integrating strategies to support the parents' wellbeing and mental health (Doyle et al., 2019, pg. 6). Here, we have demonstrated an effective, although small-scale, example of this practice for consideration.

In conclusion, this proof of concept study demonstrates that MSCC is an acceptable, feasible, and safe intervention to address the chronic stress, as well as associated mental health challenges, of parenting a child with ASD. The research design supports high advisability of further evaluation of MSCC especially within randomized controlled trials. The use of MSCC for other types of caregivers, including formal/professional caregivers and caregivers of individuals with other chronic health needs, as well as the underlying model of MSCC, warrants future exploration, using rigorous research methods and adequate and diverse samples.

Acknowledgments We are grateful for the support of the Jerry M. Lewis Mental Health Research Foundation for the funding for this project. Thanks to Lora Israelsen and Hayden Mbroh, who served as co-facilitators, as well as Jenny Kim and Emma Clark for their research assistance. We appreciate the support of the Department of Psychiatry, especially Carol Tamminga, M.D., Distinguished Chair. We thank the administration, providers, and staff at the Center for Autism and Developmental Disabilities who contributed to the success of this project and enthusiastically supported its continuation, especially Leticia Hines and Sue Schell. Most importantly, we thank the parents for their openness in participating and their time.

Author Contributions $\mathrm{KB}$ wrote the manuscript with support from RJ, SE, LH, and BW. LH performed the statistical analyses. BW administered surveys for data collection. JK and EC completed data entry.
Funding Funding for this study was provided through a grant from the Jerry M. Lewis Mental Health Research Foundation; Dallas, Texas.

\section{Declarations}

Conflict of interest Robin Jarrett, $\mathrm{PhD}$ is a paid consultant to National Institute of Mental Health and UpToDate. She has equity in Amgen, Johnson and Johnson, and Procter and Gamble. The remaining authors have no relevant financial or non-financial interests to disclose. While the medical center collects payments for the patient services provided by Dr. Bellone, Dr. Elliott, Dr. Jarrett, and Beth Warren, payments were not collected for any of the activities described in this study.

Consent to Participate Written informed consent was obtained from all individual participants included in the study prior to screening.

Ethics Approval Approval for all study procedures was obtained from the Institutional Review Board of the University of Texas Southwestern Medical Center.

\section{References}

American Psychiatric Association. (2013). Diagnostic and statistical manual of mental disorders (5th ed.). American Psychiatric Association: Arlington.

Anderson, C. (2010). "Relieving Parental Stress and Depression: How Helping Parents Helps Children.” Interactive Autism Network. Retrieved from: https://iancommunity.org/cs/articles/parental_ depression

Baer, R. A., Carmody, J., \& Hunsinger, M. (2012). Weekly change in mindfulness and perceived stress in a mindfulness-based stress reduction intervention. Journal of Clinical Psychology, 68(7), 755-765. https://doi.org/10.1002/jclp.21865

Bogels, S., Hoogstad, B., vanDun, L., de Schutter, S., \& Restifo, K. (2008). Mindfulness training for adolescents with externalizing disorders and their parents. Behavioural and Cognitive Psychotherapy, 36, 193-209. https://doi.org/10.1017/S13524658080041 90

Davis, N. O., \& Carter, A. S. (2008). Parenting stress in mothers and fathers of toddlers with autism spectrum disorders: Associations with child characteristics. Journal of Autism and Developmental Disorders, 38, 1278-1291.

Deater-Deckard, K., Chen, N., \& El Mallah, S. (2013). Parenting Stress. Oxford University Press.

de Bruin, E. I., Blom, R., \& Smit, F. (2015). MYmind: Mindfulness training for youngsters with autism spectrum disorders and their parents. Autism: The International Journal of Research and Practice, 19(8), 906-914.

Doyle, S., Chavez, S., Cohen, S., \& Morrison, S. (2019). Fostering Social and Emotional Health through Pediatric Primary Care: Common Threads to Transform Practice and Systems. Center for the Study of Social Policy. Available at: https://CSSP.org/resou rce/pspintervention-analysis-report/.

Gershon, R. C., Wagster, M. V., Hendrie, H. C., Fox, N. A., Cook, K. F., \& Nowinski, C. J. (2013). NIH toolbox for assessment of neurological and behavioral function. Neurology, 80(11 Suppl 3), S2-S6. https://doi.org/10.1212/WNL.0b013e3182872e5f

Gilmore, L., \& Cuskelly, M. (2008). Factor structure of the parenting sense of competence scale using a normative sample. Child: Care, Health, and Development, 35(1), 48-55.

Gu, J., Strauss, C., Crane, C., Barnhofer, T., Karl, A., Cavanagh, K., \& Kuyken, W. (2016). Examining the factor structure of the 39-item 
and 15-item versions of the Five-Facet Mindfulness Questionnaire before and after Mindfulness-Based Cognitive Therapy for people with recurrent depression. Psychological Assessment. https://doi. org/10.1037/pas0000263

Harris, P. A., Taylor, R., Thielke, R., Payne, J., Gonzalez, N., \& Conde, J. G. (2009). Research electronic data capture (REDCap) - A metadata-driven methodology and workflow process for providing translational research informatics support. Journal Biomedical Informatics, 42(2), 377-81.

Hayes, S. A., \& Watson, S. L. (2013). The impact of parenting stress: A meta-analysis of studies comparing the experience of parenting stress in parents of children with and without autism spectrum disorder. Journal of Autism and Developmental Disorders, 43, 629-642.

Hofmann, S. G., Sawyer, A. T., Witt, A. A., \& Oh, D. (2010). The effect of mindfulness-based therapy on anxiety and depression: A metaanalytic review. Journal of Consulting and Clinical Psychology, 78(2), 169-183. https://doi.org/10.1037/a0018555

Iadarola, S., Levato, L., Harrison, B., Smith, T., Lecavalier, L., Johnson, C., Swiezy, N., Bearss, K., \& Scahill, L. (2018). Teaching parents behavioral strategies for autism spectrum disorder (ASD): Effects on stress, strain, and competence. Journal of Autism and Developmental Disorders, 48(4), 1031-1040. https://doi.org/10. 1007/s10803-017-3339-2

Jarrett, R. B. (2018). Conceptual models of emotional states and mindful self-care within parenting a child with autism spectrum disorder. Unpublished model developed for Mindful Self-Care for Caregivers. Department of Psychiatry, University of Texas Southwestern Medical Center.

Johnston, C., \& Mash, E. J. (1989). A measure of parenting satisfaction and efficacy. Journal of Clinical Child Pychology, 18(2), 167-175.

Kabat-Zinn, J. (1990). Full catastrophe living: Using the wisdom of your body and mind to face stress, pain and illness. Delacorte.

Kabat-Zinn, J. (2013). Full catastrophe living (revised edition): Using the wisdom of your body and mind to face stress, pain, and illness. Random House Publishing Group.

Kroenke, K., Spitzer, R. L., \& Williams, J. B. (2001). The PHQ-9: Validity of a brief depression severity measure. Journal of General Internal Medicine, 16(9), 606-613. https://doi.org/10.1046/j. 1525-1497.2001.016009606.x

Kuijpers, H. J. H., Van Der Heijden, F. M. M. A., Tuinier, S., \& Verhoeven, W. M. A. (2007). Meditation-induced psychosis. Psychopathology, 40(6), 461-464. https://doi.org/10.1159/000108125

Kupst, M. J., Butt, Z., Stoney, C. M., Griffith, J. W., Salsman, J. M., Folkman, S., \& Cella, D. (2015). Assessing stress and self-efficacy for the NIH toolbox for neurological and behavioral function. Anxiety, Stress, and Coping, 28(5), 531-544.

Lecavalier, L., Leone, S., \& Wiltz, J. (2006). The impact of behavior problems on caregiver stress in young people with autism spectrum disorders. Journal of Intellectual Disability Research, 50(3), 172-183.

Leslie, L. K., Mehus, C. J., Hawkins, J. D., Boat, T., McCabe, M. A., Barkin, S., Perrin, E. C., Metzler, C. W., Prado, G., Tait, V. F., Brown, R., \& Beardslee, W. (2016). Primary health care: Potential home for family-focused preventive interventions. American Journal of Preventive Medicine, 51(4-2), S106-S118. https://doi. org/10.1016/j.amepre.2016.05.014

Lumpkin, M., \& Jarrett, R. B. (2015). Mindful self-care for caregivers (MSCC): A guide for group facilitators. Unpublished treatment manual. Department of Psychiatry. The University of Texas Southwestern Medical Center. Dallas, TX.

Mallinckrodt, C. H., Kaiser, C. J., Watkin, J. G., Molenberghs, G., \& Carroll, R. J. (2004). The effect of correlation structure on treatment contrasts estimated from incomplete clinical trial data with likelihood-based repeated measures compared with last observation carried forward ANOVA. Clinical Trials, 1, 477-489.
Mathew, N. E., Burton, K. L. O., Schierbeek, A., Crncec, R., Walter, A., \& Eapen, V. (2019). Parenting preschoolers with autism: Socioeconomic influences on wellbeing and sense of competence. World Journal of Psychiatry, 9(2), 30-46. https://doi.org/10.5498/ wjp.v9.i2.30

National Alliance on Mental Illness. (2020). COVID-19 Resource and Information Guide. https://www.nami.org/covid-19-guide

Osborne, L. A., McHugh, L., Saunders, J., \& Reed, P. (2008). Parenting stress reduces the effectiveness of early teaching interventions for autistic spectrum disorders. Journal of Autism and Developmental Disorders, 38, 1092-1103. https://doi.org/10.1007/ s10803-007-0497-7

Pordes, E., Gordon, J., Sanders, L. M., \& Cohen, E. (2018). Models of care delivery for children with medical complexity. Pediatrics, 141, S212. https://doi.org/10.1542/peds.2017-1284F

Ridderinkhof, A., deBruin, E. I., Bogels, S., \& Blom, R. (2018). Mindfulness-based programs for children with autism spectrum disorders and their parents: Direct and long-term improvements. Mindfulness, 9(3), 773-791. https://doi.org/10.1007/ s12671-017-0815-x

Salsman, J. M., Butt, Z., Pilkonis, P. A., Cyranowski, J. M., Zill, N., Hendrie, H. C., Kupst, M. J., Kelly, M. A., Bode, R. K., Choi, S. W., Lai, J. S., Griffith, J. W., Stoney, C. M., Brouwers, P., Knox, S. S., \& Cella, D. (2013). Emotion assessment using the NIH Toolbox. Neurology, 80(11 Suppl 3), S76-86.

Schieve, L. A., Blumberg, S. J., Rice, C., Visser, S. N., \& Boyle, C. (2007). The relationship between autism and parenting stress. Pediatrics, 119, S114-S121.

Schlosser, M., Sparby, T., Vörös, S., Jones, R., \& Marchant, N. L. (2019). Unpleasant meditation-related experiences in regular meditators: Prevalence, predictors, and conceptual considerations. PLoS ONE, 14(5), e0216643. https://doi.org/10.1371/journ al.pone. 0216643

Sharma, P., Mahapatra, A., \& Gupta, R. (2019). Meditation-induced psychosis: A narrative review and individual patient data analysis. Irish Journal of Psychological Medicine. https://doi.org/10.1017/ ipm.2019.47

Sharpley, C. F., Bitsika, V., \& Efremidis, B. (1997). Influence of gender, parental health, and perceived expertise of assistance upon stress, anxiety, and depression among parents of children with autism. Journal of Intellectual and Developmental Disability, 22, $19-28$.

Sheehan, D. (2016). Mini international neuropsychiatric interview for attention defici/hyperactivity disorder studies (adults), English version 7.0.2 for DSM-5. Retrieved from: https://harmresearch. org/index.php/product/mini-international-neuropsychiatric-inter view-mini-7-0-2-13/

Sheehan, D. V., Lecrubier, Y., Harnett-Sheehan, K., Amorim, P., Janavs, J., Weiller, E., Hergueta, T., Baker, R., \& Dunbar, G. (1998). The mini international neuropsychiatric interview (M.I.N.I.): The development and validation of a structured diagnostic psychiatric interview. J Clin Psychiatry, 59(suppl 20), 22-33.

Singh, N., Lancioni, G., Winton, A., Fisher, B., Wahler, R., Mcaleavey, K., Singh, J., \& Sabaawi, M. (2006). Mindful parenting decreases aggression, noncompliance, and self-injury in children withautism. Journal of Emotional and Behavioral Disorders., 14, 169-177.

Teasdale, J. D., Segal, Z., \& Williams, J. M. (1995). How does cognitive therapy prevent depressive relapse and why should attention control (mindfulness) training help? Behavioral Research and Therapy, 33(1), 25-39.

Tonge, B., Brereton, A., Kiomall, M., Mackinnon, A., \& Rinehart, N. J. (2014). A randomized group comparison controlled trial of 'preschoolers with autism': A parent education and skills training 
intervention for young children with autistic disorder. Autism, 18(2), 166-177.

Veehof, M. M., Trompetter, H. R., Bohlmeijer, E. T., \& Schreurs, K. M. G. (2016). Acceptance-and mindfulness-based interventions for the treatment of chronic pain: A meta-analytic review. Cognitive Behaviour Therapy, 45(1), 5-31.
World Health Organization (2002). Gender and Mental Health. Available at: https://www.who.int/gender/other_health/genderMH.pdf

Publisher's Note Springer Nature remains neutral with regard to jurisdictional claims in published maps and institutional affiliations. 